\title{
Marfan syndrome with a giant noncoronary sinus of Valsalva aneurysm
}

\author{
Sahin Avsar, Sennur Unal Dayi, Adnan Kaya, Ibrahim Levent Ozmutlu, Altug Osken \\ Dr. Siyami Ersek Cardiovascular Surgery Hospital, Istanbul, Turkey
}
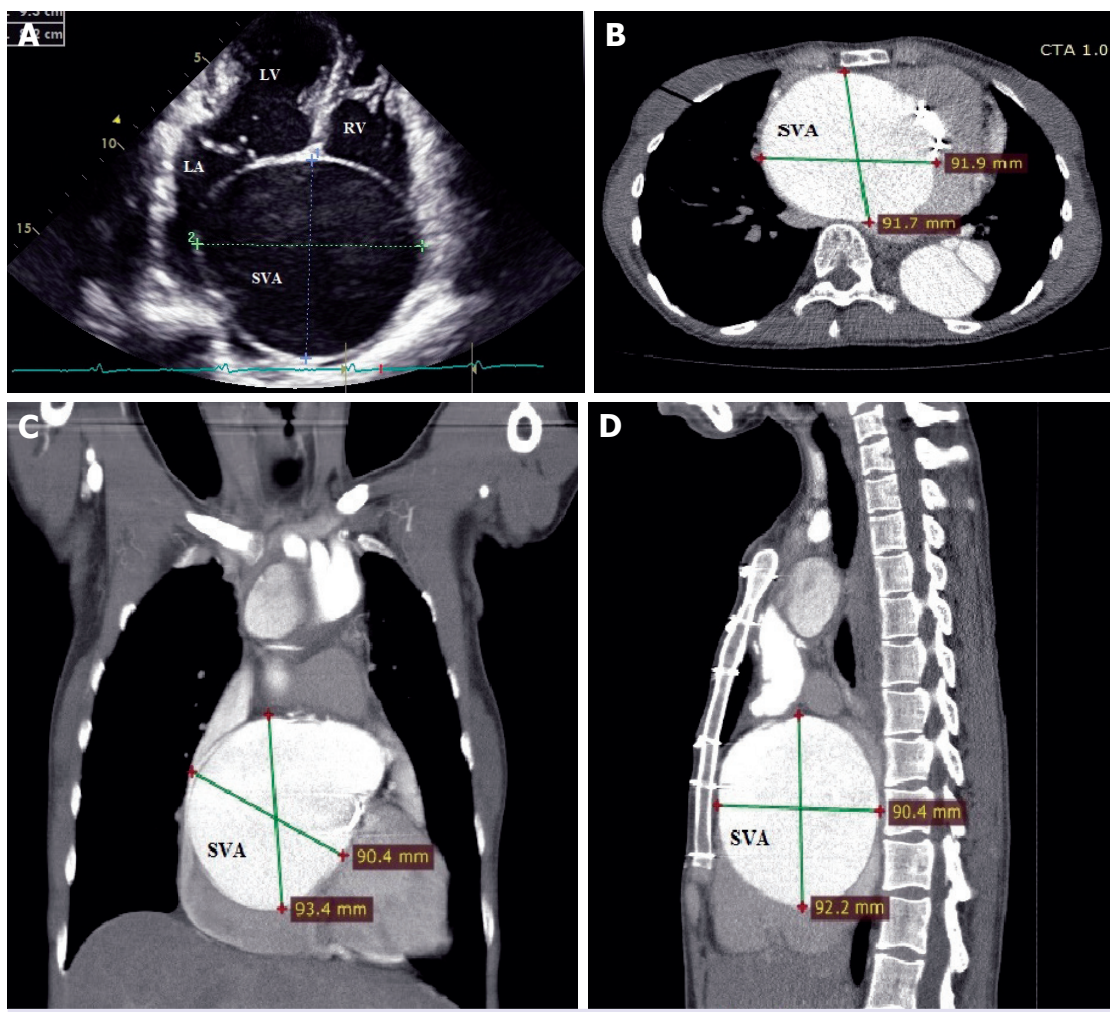

FIGURE 1. (A) Transthoracic echocardiogram in the apical four-chamber view showing a giant noncoronary sinus of Valsalva aneurysm (SVA). (B, C, D) Cardiac computed tomography images. (B) Axial image showing noncoronary SVA compressing all pulmonary veins (PV) and the LA. The dissection flap in the descending thoracic aorta is also seen between the false (FL) and true (TL) lumens. (C) Coronal image showing noncoronary SVA compressing the RA. Arrow indicates the mechanical aortic valve. (D) Sagittal image showing noncoronary SVA. LV: Left ventricle; RV: Right ventricle.
Once presented in 2011 by Dayi SU et al. our case is a 30-year-old woman with Marfan syndrome who had undergone aortic valve replacement due to severe aortic regurgitation secondary to dilatation of the aortic root in 2000 and supracoronary aortic replacement due to Stanford type A dissection in 2002 with subsequent dilatation of sinus of Valsalva. When described by Dayi SU et al. the size of the aneurysm was noted to be $6 \times 6.5 \times 6.5 \mathrm{~cm}$ in dimensions and it was formed from noncoronary sinus of Valsalva. At this time conservative follow - up was found to be the appropriate therapy because high risk of redo surgery. Three years later the same patient was observed in echocardiography laboratory with a rapid progression of the same aneurysm of sinus of Valsalva to $8.3 \times 9.3 \times 8.2 \mathrm{~cm}$ in size (Figure 1A). Moderate mitral and tricuspid regurgitation with prolapse of valves and normal function of mechanical aortic valve were observed. Computed tomography angiography also confirmed enlargement of the aneurysm to $91.9 \times 91.8$ in size (Figures $1 B, C$, and D). Herein, we would like to draw attention to the clinical presentation and progression of Marfan syndrome. 